\title{
Open Stack Cloud Tuning and Network Acceleration for HPC and Datacenter Intelligent Applications
}

\author{
Shashi Kant Gupta ${ }^{1}$ Ashish Kumar Pandey ${ }^{2}$ \\ ${ }^{1}$ Assistant Professor, CSE Department, Pranveer Singh Institute of Technology, Kanpur, Uttar Pradesh- 209305
${ }^{2}$ Assistant Professor, CS\&E Department, IET, Dr. R.M. LAvadh University, Ayodhya, UP, India
}

To Cite this Article

Shashi Kant Gupta and Ashish Kumar Pandey, "Open Stack Cloud Tuning and Network Acceleration for HPC and Datacenter Intelligent Applications", International Journal for Modern Trends in Science and Technology, 6(8S): 06-12, 2020.

\section{Article Info}

Received on 16-July-2020, Revised on 15-August-2020, Accepted on 25-August-2020, Published on 28-August-2020.

\section{ABSTRACT}

HPC is scarcely attempted known clouds as a consequence of inefficient and slow Inter VM interaction on exactly comparable server and big latency between remote products. It was converted by launch of ivshmem, a PCI product reliant shared remembering between VMs on precisely exactly the same server, but unfortunately, this particular mechanism improved turning into busted off of with Linux update barely any yrs refunded. We've restored this particular shared recalling framework subsequently created, as a result of the point during initial likelihood, complete cloud integration making use of current editions of OpenStack, Linux, QEMU, MPICH. and libvirt Also, the analyses of various variables influencing every single TCP/IP and in addition ivshmem interaction is actually supplied along with tuning methods which may considerably improve general entire performance. Multi-Tenant media as well as cloud virtualization provide Infrastructure as a system (IaaS) suppliers a new and innovative technique to offer on demand solutions to the consumers of theirs, for instance simple provisioning of entirely new applications in addition to far better supply efficiency in addition to scalability. Nevertheless, current details comprehensive wise uses call for much better processors, greater bandwidth in addition to lower latency advertising plan. Therefore, as to boost the general functionality of computing in addition to social network expertise, as well as reduce the overhead of a software program virtualization, we recommend a new info facility method layout based on OpenStack. Specifically, we chart the OpenStack network offerings on the hardware switch and also make use of hardware accelerated L2 switch and also L3 routing to solve the software limits, and likewise attain program as scalability in addition to flexibility. We model the prototype process of ours over the Arista Software-Defined-Networking (SDN) switch and present a quick piece of a software application which abstracts the system level which decouples OpenStack with the real physical society infrastructure, thus providing vendor freedom. The formula of ours shows improved cloud scaling in addition to local community efficiency via only one communication item to control each vendors' treatments to the info facility.

KEYWORDS: OpenStack; cloud computing; high performance computing; cloud tuning, Arista, EOS, Neutron, LinuxBridge, Cloud Computing, Cloud Virtualization 


\section{INTRODUCTION}

Nowadays, Cloud Computing may be the dominating common objective computing paradigm, along with OpenStack [1] is believed basically the most favoured accessible supply cloud OS for particular clouds. Regrettably, High-Performance-Computing (HPC) inside a cloud wasn't apt in only yesteryear, as a consequence of the sizable overhead for inter VM correspondence throughout one server as well as between servers too, since it'd been discovered for instance by [2], [3]. Nevertheless, using the entrance of a remake of ivshmem [4], a shared keeping in mind among VMs on exactly comparable server started to regularly be attainable once more, therefore creating HPC inside technique reasonable for an OpenStack cloud.

Due to this specific newspaper, a set of OpenStack tuning techniques are actually mentioned augment the choices computer users have for OpenStack was discovered by HPC, so long as MPICH [5] is actually interested. For which goal that is specific, we investigated the scenario which each MPICH therapy is actually allocated to just one $\mathrm{VM}$ in addition to evaluated the inter VM interaction on exactly comparable server with the usage of the telephone refers to as MPI_PUT for info exchange as well as MPI_WIN_LOCK for info synchronization. Each \& every telephone refers to as was wrapped by us close to the first MPICH telephone refers to as of exactly related brand to hold the capability to have the capability to the office the ivshmem remake identified OpenStack. We are going to exhibit within the following several effectiveness tuning techniques for HPC found OpenStack via ivshmem, and furthermore for that classical inter VM interaction via TCP/IP that's seated on Neutron's [6] Open vSwitch [7] building. Through the steps of ours, inter VM bandwidth along with latency could be improved by means of a point to think about of nearly as 6 , from quite a few harmful scenarios to better scenario, since the complete functionality dimensions of ours show. The net nowadays is actually information pressed, with approximately ninety exabytes of information realizing each month within the type of video clip fasteners, photographs, combined with a lot of other enriched compound [8]. The necessity for excellent virtualized information middle device is soaring. Large and small companies are actually seeing the cloud having a huge velocity, with about 175 billion cash of earnings produced from public cloud therapies within the time of year 2016 [9].
The current information center page layout has transformed to a software program defined neighbourhood society process with virtualization building the crux of all of the recently commissioned information facilities. Velocity, overall functionality, after which ease of access have an inclination to perform as the essential requirements of the strategy. It's essential to pick out a good society framework that fulfils every one of the specs, with hardly any sale price, fast deployment, simple management, after which scalability. Nevertheless, the device overall performance on the existing cloud is not on exactly the very same quality as what of regular high-performance computing (HPC), which usually utilizes InfiniBand for the system [10]. Nevertheless, classy HPC grids coupled with clusters can't fine tune to successful workloads or perhaps maybe perhaps allot property to concurrent multi-tenant purposes [11], [12], [13]. Software program described networks paradigm can assist resolve the issue by isolating degree $2 / 3$ functionalities within tenant networks by making use of Openv Switch (OVS) or maybe Linux bridges. Method modifications produce an important component of each virtualized computing increase job. They supply local society entry for Virtual clothing airers [14]. Most probably the numerous widely used virtual switch put in position, Openv Switch (OVS), is extremely used in cloud computing os's as OpenStack. Nevertheless, this particular remedy doesn't be competitive with typical general functionality number of regular press as fallen through devoted local society hardware. This's due to the many dependencies of package deal processing within the software program, which substantially hinders general entire performance.

\section{SYSTEM DESIGN}

Developing an individual, hybrid or public OpenStack cloud calls for physical and virtual society infrastructure which is resilient, agile, and programmable. We suggest providing an incredibly scalable as well as automated cloud infrastructure for an OpenStack location by way of a fast-small bit of a software program. By utilizing our suggested Enhanced Switch Offloading for OpenStack Network (ESOO), purchasers have the capability to considerably speed up business knowledge, mitigate purposeful intricacy and lower bills. The framework on the suggested "ESOO" of ours is actually realized with Fig. 1. The ESOO framework 
of ours concentrates on boosting the OpenStack os normal performance by altering the LB/OVS bundle deal flipping with hardware reliant transferring much more than. While carrying out this, this particular brand-new environment won't impact the independence on the unit hands as well as wrists completely free functioning or maybe provide a handful of compatibility problems. The framework of ours offers a total point of view on the unit (topology) coupled with its consistent and current state round the fundamental hardware switch. When an OpenStack unique capability a couple of social media connected working (create/update/delete/read on community that is local, subnet along with port resources), the Neutron server gets the petition, techniques by the very same on the set-up WordPress plugin and in addition tends to come up with the appropriate change on the DB. The ESOO of ours in addition leverages the Intel's Data Plane Development Kit (DPDK) [eight] to undertake the computational overhead when accessing the unit operator pc user interface flash imagination flash memory card (NIC) with the Hypervisor. If the GPU could be received to the server along with users' software package requires GPU velocity, the ESOO framework of ours is going to setting up the GPU pass through because of this particular tenant instantly. By executing hardware linked setup then set up instantly, members are actually proficient to buy the best overall performance outside the information facility in just a transparent manner. For TCP/IP tuning in addition to moreover, the consequent chapters, we apply OpenStack Juno, Ubuntu 16.04 as website visitor OS, CentOS 7.1 as range that is wide OS, libvirt 2.0, QEMU 2.9.50, MPICH 3.2 along with virtio 1.1 .1 as a software program atmosphere. Additionally, we sent information through one MPI pastime on the various extra, while different the dimensions of its through 22 to 220 bytes. The transmission was attained by one sided MPI_PUT with the traditional framework MPICH Nemesis sock channel. Nevertheless, as a consequence of the basic fact that there's typically barely any discussed keeping in mind between several VMs, quite likely on exactly comparable server, MPIC emulates the general performance by TCP/IP interaction, generating information packets forth and back which have discussed variables.

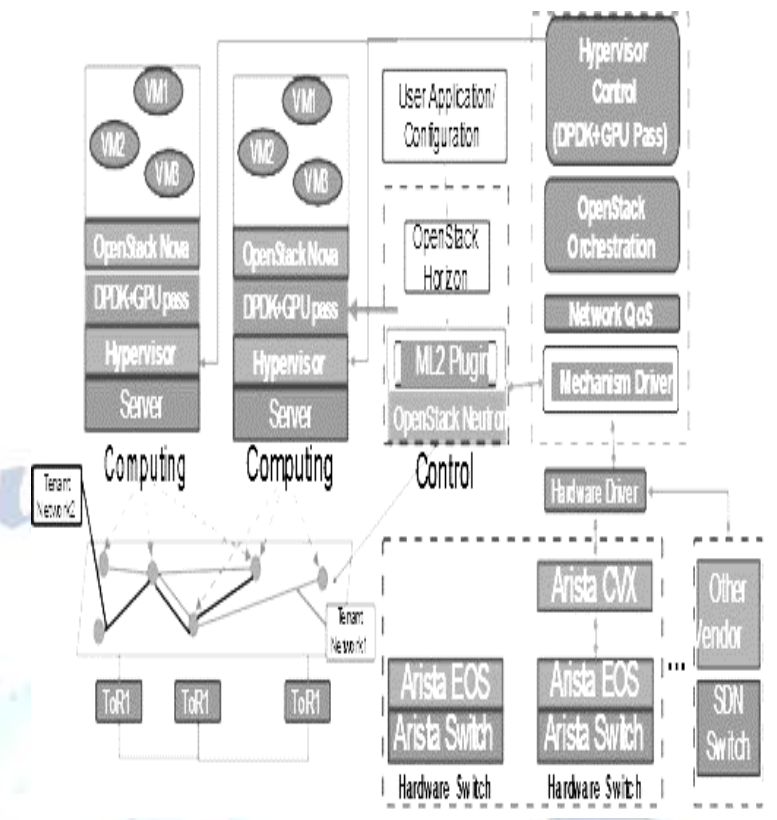

Figure 1. System Overview

\section{STATE OF THE ART IN INTER-VM COMMUNICATION IN OPENSTACK}

As any type of sort of cloud, OpenStack is actually a sent-out method, if the cloud is actually positioned within an equivalent rack and even possibly even inside an equivalent computing facility where TCP/IP wouldn't be required, since L2 altering may be enough. By checking out [7], we may present an obstruct diagram within the ensuing uses overhead (Fig. 2) for the circumstance which 2 MPICH techniques are actually carried out by 2 VMs over the identical server. In accordance with Fig. 2, any type of kind of information frame has moving 2 events coming out of the following stages: TCP/IP stack, printer car proprietor, virtual society operator pc user interface, qbr Linux Bridge, Open vSwitch (OVS) Integration Bridge, OVS VLAN Bridge, genuine actual bodily Ethernet Interface, physical Ethernet Switch. No matter the stage which OVS is actually a part of OpenStack's Neutron technique program as well as specific port configurations might perhaps provide undesirable volume of intermediate interfaces, it nevertheless creates a great deal of overhead. Combined with the VM correspondence overhead, the quick effect is actually an undesirable small HPC success. 


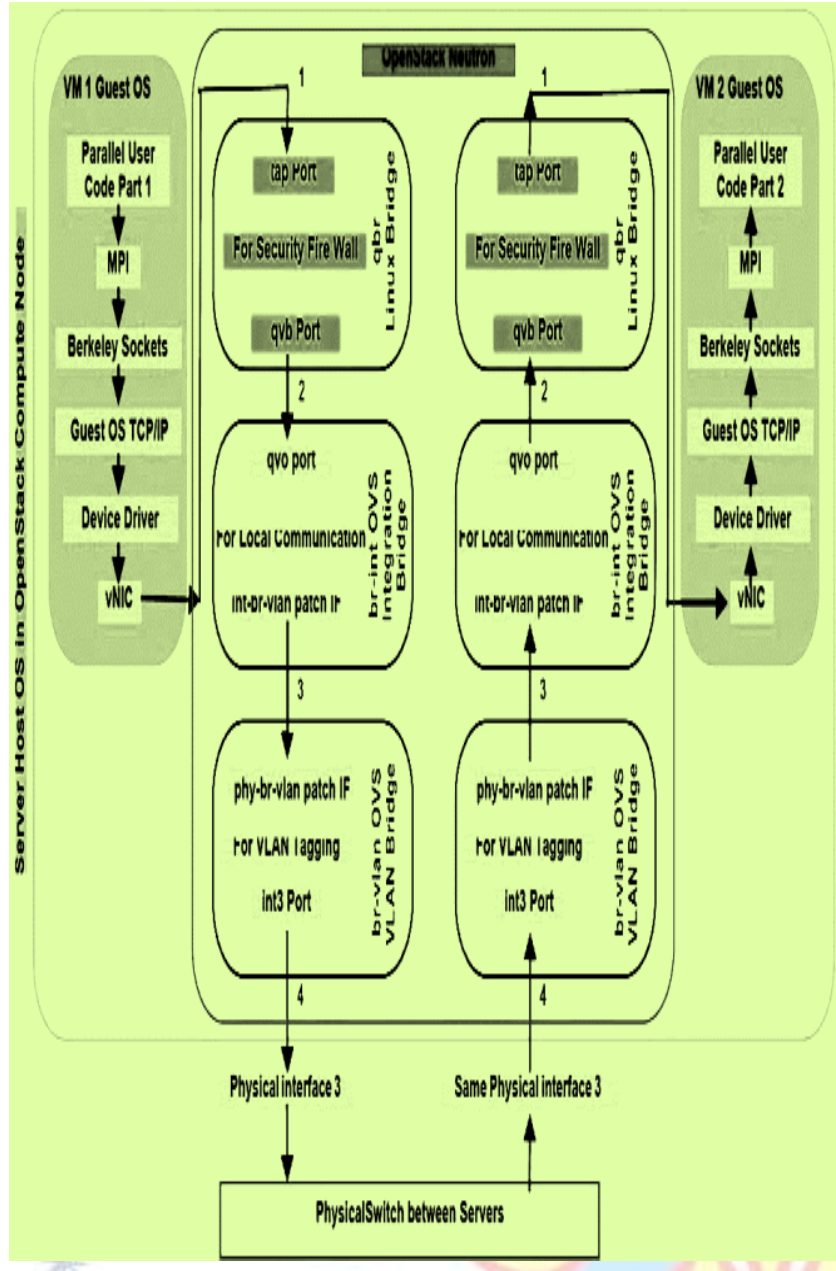

Figure 2. Overhead of Software in OpenStack for I-VM communication. 1,2,3 =Ethernet-Data Frames, 4=VLAN-tagged Ethernet Frames, OVS = Open vSwitch.

\section{TUNING THE CLASICAL TCP/IP INTER-VM COMMUNICATION}

For TCP/IP tuning in addition to moreover, the consequent chapters, we apply OpenStack Juno, Ubuntu 16.04 as website visitor OS, CentOS 7.1 as range that is wide OS, libvirt 2.0, QEMU 2.9.50, MPICH 3.2 along with virtio 1.1 .1 as a software program atmosphere. Additionally, we sent information through one MPI pastime on the various extra, while different the dimensions of its through 22 to 220 bytes. The transmission was attained by one sided MPI_PUT with the traditional framework MPICH Nemesis sock channel. Nevertheless, as a consequence of the basic fact that there's typically barely any discussed keeping in mind between several VMs, quite likely on exactly comparable server, MPICH emulates the general performance by TCP/IP interaction, generating information packets forth and back which have discussed variables.

\section{IVSHMEM}

Ivshmem is actually a virtual PCI iteminside a website visitor OS that's imitated by KVM/QEMU. It establishes a Linux/POSIX shared mind (SHM) between the VM along with its broad range OS. Ivshmem hence permits zero message VM-to-Host interaction together with the different other means round, together with that is somewhat effective with well worth to latency and bandwidth, since barely any inner information buffer is actually available. Ivshmem may likewise be utilized for inter VM interaction coupled with the broad range OS SHM as intermediate undertaking. Ivshmem is actually utilized by mapping its virtual PCI unit creativity with the range that is wide OS SHM. This's possible, since the human brain is actually imitated by QEMU becoming an information activity inside of itself also, since a choice of QEMUs is able to talk to one another discovered broad range OS.

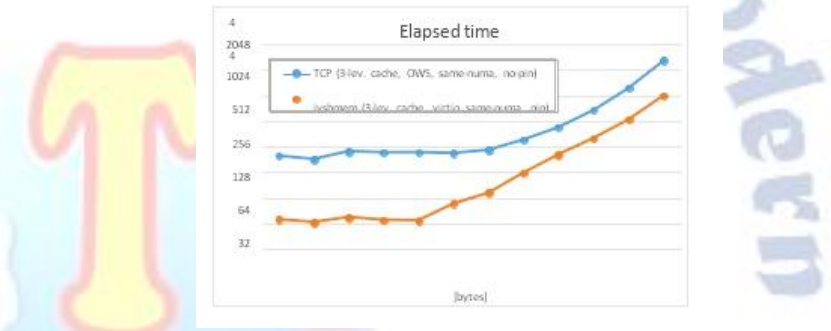

Figure 3.TCP and ivshmem-based inter-VM communication Elapsed times

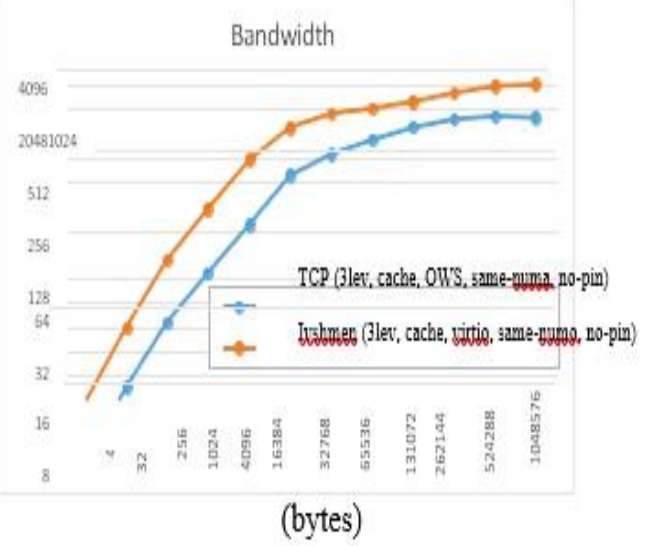

Figure 4. TCP and ivshmem-based inter-VM communication Bandwidth

Last but not least, although not least, immediate comparability of normal TCP/IP cloud (OVS) performance combined with the ivshmem integration of ours is actually depicted within Fig. 3 along with Fig. 4, for Elapsed Bandwidth and Time respectively. We've intentionally owned or even operated very same NUMA place for 
width and length, since NUMA allocation is actually achieved arbitrarily by OpenStack scheduler combined with each illustration advancement might well meet up with an assortment of mind region. For which scaled down length as well as width marketing communications, when synchronization is actually dominating interaction, typical efficiency distinction is actually part of 6 all around favour of ivshmem. With additional improvement of note obstruct sizing, information flow gets primary contributor to accomplish correspondence time and moreover, the difference drops to a part of 3 , mainly because the very best marketing as well as product sales communications. This's amazing impact, thinking about the point that NUMA tuning wasn't used, because of arbitrary qualities of OpenStack scheduler.

\section{Vi. Cloud as well as Network Topology}

We suggest a prototype information facility seem coupled with the next 4 components: one) Main Server: Runs primary OpenStack expertise, and also performs whether the Horizon dash board for people to handle circumstances. The main Server is actually in addition the con troller node, together with that is the main hub for every one of the instructions performed. Numerous OpenStack servers for example Keystone for authentication, Glance for picture storage room region, neutron for social networking, in addition to a selection of others is actually maintained throughout the controller node. This particular node allows the end user to possess interaction coupled with the various supported APIs \&amp; moreover, the dash board. Many other elements like Database, Block Storage, and also Object Storage could be split into other impartial nodes. two) Arista Switch: 2 7050x EOS modifications are utilized a top part of Rack (TOR) modifications, too as a medialink wireless router for various networks inside OpenStack. three) Rack Servers: 2 Dell R630 servers with powerful processor and high memory are popular as computing nodes for AI applications. four) CVX Server: One server is actually functioning vEOS to receive the task finished because the CVX Server. Simply because the CVX Client is actually operated from the hardware switch. The CVX Server is needed when the middleware for talking between physical EOS modifications coupled with OpenStack Neutron by EOS API (eAPI). The CVX
Server can become educated on community growth that is brand new coming from Neutron, as well as disperse the info to CVX Client. five) Provider host: one particular computing node (computer) as the gateway is actually utilized to use the provider activity for offering with cases in addition to tests ease of access. The prototype method earth is displayed with Fig. five.

vendor-independent design \& style attainable. With this particular paper, we use Arista 's SDN switch as a good example. The OpenStack printer driver of ours utilizes Arista's EOS API (eAPI) to consult with Arista's Cloud Vision eXchange (CVX), with the entire perspective of all the attached Arista improvements. Within the circumstance of disappointment, CVX has the ability to resync the state of its. The server furthermore is going to keep a topology of accessible real physical alterations \&amp; hosting businesses, along with OpenStack scenarios. Using CVX simplifies the interaction combined with the hardware switch. For example, only one user interface is actually necessary for Arista's switch or perhaps various other user interface to connect up to other vendors' hardware without linking every single switch individually. To reduce the overhead when accessing the ca pc user interface flash memory card (NIC) in addition to GPU in the VM, we put into action the Intel DPDK as well as GPU pass through to the hypervisor to installation the quick info track.

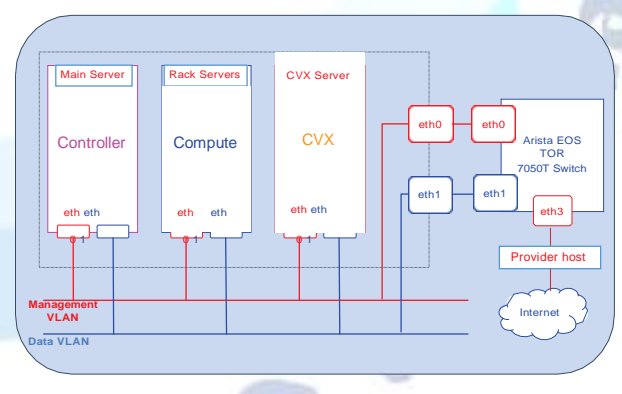

Figure 5. Prototype private cloud's system topology

\section{QuAlity OF SERVICE (QOS) TEST}

Therefore, as to check out the quality of Service (QoS) and also compute the outcome on the QoS from some other prospects, we've a more advanced topology with a number of nodes and additionally a bunch of website traffic patterns. The examination topology of ours includes six nodes as follows:

1) Client one in addition to Client two are actually positioned around Net One with QoS 
policy put on to, in addition to site traffic is actually mailed by them to Server- and Server- 1 two, respectively; 2) The Server one is actually situated outside the current datacenter and in addition might be seen out of the provider process along with medialink wireless medialink wireless router "EOS R2"; 3) The Server 2 is actually utilized in Net 2 along with associated combined with the customer by exactly comparable medialink wireless router "EOS R1"; "Flooder" is actually situated on Net 4 with 2 parallel 5Gbps visitors to Hypervisor-1 and Server-1, respectively. "Flooder" is actually employed by us to model more society traffics which make use of the accessible bandwidth. Therefore, as to constantly keep the QoS policy, the Open Stack Neutron is going to need to dynamically allot the unit bandwidth for all those networks/instances. This specific treatment is actually used by us to mimic a helpful datacenter technique community in addition to check out the practical use on the ESOO pattern of ours below the QoS circumstances.

We place into motion one QoS policy with sites one for the same Client-2 and Client- 1 inside a single team. The QoS policy comprises of two rules: 1) the very best bandwidth of $4 \mathrm{Gbps}$ along with 2) a really least bandwidth of $2 \mathrm{Gbps}$. If the QoS policy is actually used, the OpenStack Neutron assures the technique is going to have at least $2 \mathrm{Gbps}$ for every ports / instance it links to and won't look at probably a largest of $4 \mathrm{Gbps}$. To read the useful functionality influence with the QoS policy on Client-2 and Client-1, we created a 4 stage benchmark visitors' format with thirty secs for each and every point. For Client one, traffic stage you're four Gbps; stage two is actually three Gbps, stage three is actually four Gbps, along with point four is actually two Gbps, i.e., a "4-3-4-2 Gbps" design and design. For Client sandal, the site traffic stage you're two Gbps, stage two is actually three Gbps, stage three is actually three Gbps, along with point four is actually four Gbps, i.e., a "2-3-3-4 Gbps" design and design. The flooder has 2 parallel links flooding $5 \mathrm{Gbps}$ website traffic out there (ten Gbps within) which is done. We completed the very same examination utilizing the ESOO put in position and also OpenStack LinuxBridge/vRouter choose to throw set up as well as opposed the variants to come down with general entire performance.
To attain probably a greatest $10 \mathrm{Gbps}$ site link someplace between real actual physical changes $\&$ servers, the bandwidth is actually delivered amongst instances and networks. Operating our proposed' ESOO' technique, the vast majority of web hosting companies below QoS policy are actually sure the positive 2Gbps minimum bandwidth along with 4Gbps optimum bandwidth as found with Fig. 6a. The OpenStack Neutron assures the QoS policy along with barely any instance/network is able to occupy all of the bandwidth. The Neutron QoS design slices lower regarding the Flooders' car traffic bandwidth to create certain the QoS policy of Client-2 and Client-1 below the optimum 10Gbps website hyperlink restriction. The QoS results in the LinuxBridge/vRouter created are actually discovered with Fig. $6 \mathrm{~b}$. Since the bandwidth outcomes of Client- and Client- 1 two, it may be discovered that the bandwidth below the QoS policy can't make sure as a result of system borders. The accessible bandwidth pool which shared among almost all network/instances is under $3 \mathrm{Gbps}$. Whenever the guests to the technique is considerably higher in comparison to the cap, the Service Level Agreement (SLA) can't be attained also every single example in the QoS policy could conveniently merely have a 0.5 Gbps bandwidth.

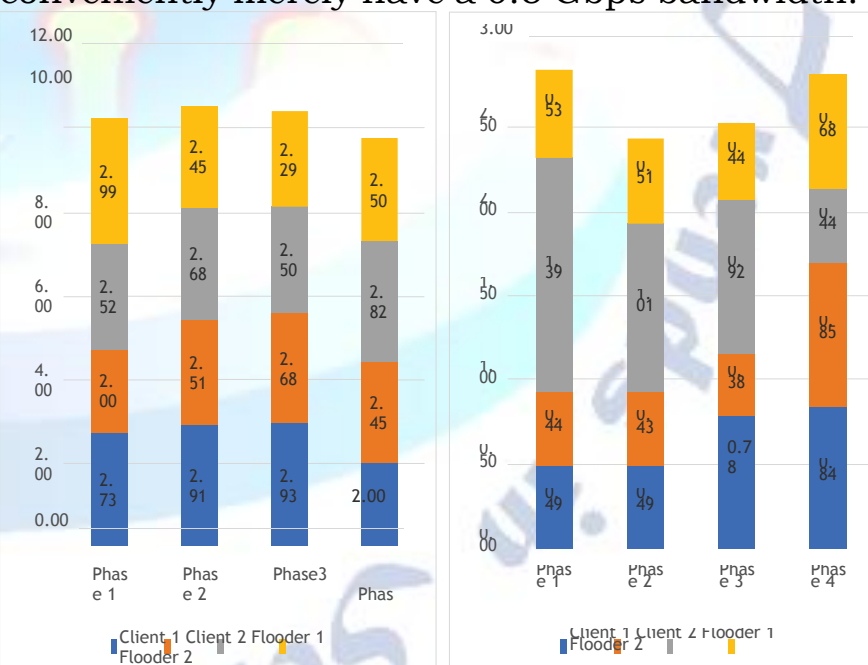

Figure: 6 (a) Figure: 6 (b)

\section{CONCLUSION}

The OpenStack technique formulation offers a selection of methods for administrators to orchestrate the cloud atmosphere of theirs. Due to this specific paper, we show a very helpful method to speed up the ca performance with the OpenStack for info complete smart requirements. The suggested ESOO framework 
of ours may be of assistance automated provisioning multi-tenant networks \& fully make use of the capabilities on the existing hardware (i.e., GPU, NIC, together with SDN Switch) without any re quiring a lot more hardware accelerators. The experimental results of ours show that the formula of ours has the capability to run the hardware on the boundaries of its (i.e., 10Gbps nearby society throughput) underneath an assortment of topology. The solution of ours aids within orchestrating the private cloud through an incredibly integrated three stage share of program while ensuring scalability, flexibility, reliability, and then working which are forced to accelerate info complete uses. High-Performance-Computing inside a cloud wasn't apt in only yesteryear, as a consequence of the sizable overhead made throughout inter VM correspondence throughout one server and also for that certain primary reason within in between remote products. It was converted with the look of a remake of ivshmem, that is a genuine shared keeping in mind among VMs on exactly equivalent large range server. As on the list of major outcomes, we created it very simple for just about any very first time to use the ivshmem remake within an OpenStack reliant private cloud. Additionally, we introduced a few of tuning techniques, for example appropriate NUMA allocation as well as CPU pinning \&amp; for that reason, adapted ivshmem to attempt to do better. In addition, we supplied advantageous steps for TCP/IP reliant emulation of genuine real bodily discussed recalling, that are degree 3 caching afterward virtio very compared to Neutron's Open vSwitch. Just about all tactics were certainly analysed or perhaps maybe even in instead of one another by width and length, indicating that the most effective ivshmem circumstance is no less than 3 instances as fast as the very best TCP/IP circumstances. Just about all length as well as width have been made up of the wrapper variants of ours of MPICH's MPI_PUT for info MPI_WIN_LOCK as well as exchange for shared mind synchronization.

\section{REFERENCES}

[1] Open source software for creating private and public clouds, https://www.openstack.org/

[2] R. Ledyayev, H. Richter, High Performance Computing in a Cloud Using OpenStack, The Fifth International Conference on Cloud Computing, GRIDs, and Virtualization, CLOUD COMPUTING 2014, http://www.iaria.org/conferences2014/CLOUDCOMPUTI NG14.html, Venice, Italy, May 25 - 29, 2014.
[3] H. Richter, About the Suitability of Clouds in High-Performance Computing, Proc. Sixth International Conference on Computer Science and Information Technology (CCSIT 2016), Journal Computer Science and Information Technology (CC\&IT), Volume 6, Number 1, January 2016, pp. 23-33, Volume Editors: Jan Zizka, Dhinaharan Nagamalai, ISBN:978-1-921987-45-8, DOI:10.5121/csit.2016.60103,

http://airccj.org/CSCP/vol6/csit64803.pdf, AIRCC Publishing Cooperation, Zurich, Switzerland, January 02-03, 2016

[4] P. Ivanovic, H. Richter, Performance Analysis of ivshmem for High- Performance Computing in Virtual Machines, Proc. 2nd International Conference on Virtualization Application and Technology (ICVAT 2017), Shenzhen, China, Nov.17-19, 2017.

[5] A. Amer, P. Balaji, W. Bland, W. Gropp, R. Latham, H. Lu, MPICH User's Guide, Version 3.2, Mathematics and Computer Science Division - Argonne National Laboratory, Nov.11, 2015

[6] Introduction to OpenStack Networking (neutron), https://docs.openstack.org/liberty/networking-guide/intr o-os- networking.html

[7] Open vSwitch in OpenStack, https://docs.openstack.org/liberty/networking-guide/sce nario-classic- ovs.html

[8] [8] The zettabyte era trends and analysis. [Online]. Available:

http://www.cisco.com/c/en/us/solutions/collateral/serv ice-

provider/visual-networking-index-vni/vni-hyperconnectivi ty- wp.html

[9] Facts and statistics about cloud computing. [Online]. Available:

https://www.statista.com/topics/1695/cloud-computing 1

[10] N. S. Islam, M.Rahman, J.Jose, R.Rajachandrasekar, H. Wang, H. Subramoni, C. Murthy, and D. K. Panda, "High performance rdma-based design of hdfs over infiniband," in Proceedings of the International Conference on High Performance Computing, Networking, Storage and Analysis. IEEE Computer Society Press, 2012, p. 35.

[11] I. Foster, Y. Zhao, I. Raicu, and S. Lu, "Cloud computing and grid computing 360-degree compared," in Grid Computing Environments Workshop, 2008. GCE'08. Ieee, 2008, pp. 1- 10 .

[12] J. Jose, M. Li, X. Lu, K. C. Kandalla, M. D. Arnold, and D. K. Panda, "Sr-iov support for virtualization on infinibandclus- ters: Early experience," in Cluster, Cloud and Grid Computing (CCGrid), 2013 13th IEEE/ACM International Symposium on. IEEE, 2013, pp. 385-392.

[13] Q. He, S. Zhou, B. Kobler, D. Duffy, and T. McGlynn, "Case study for running hpc applications in public clouds," in Proceedings of the 19th ACM International Symposium on High Performance Distributed Computing. ACM, 2010, pp. 395-401.

[14] B. Pfaff, J. Pettit, T. Koponen, E. J. Jackson, A. Zhou, J. Rajahalme, J. Gross, A. Wang, J. Stringer, P. Shelar et al., "The design and implementation of open vswitch." in NSDI, 2015, pp. 117-130. 